\title{
Status of the LHCf experiment and future prospects
}

\author{
Yoshitaka Itow for the LHCf collaboration ${ }^{* \dagger}$ \\ STE lab/KMI, Nagoya University \\ E-mail: itowdstelab.nagova-u.ac.jp
}

The Large Hadron Collider forward (LHCf) experiment is a special purpose experiment dedicated to measure the production of neutral particles at the very forward region of the LHC. The data are useful to verify hadronic interaction that influence the development of an air shower. Until now data have been obtained for $\mathrm{p}-\mathrm{p}$ collisions at $\sqrt{s}=0.9$ and $7 \mathrm{TeV}$ and $\mathrm{p}-\mathrm{Pb}$ collisions at $\sqrt{s}=$ $5.02 \mathrm{TeV} / n$. Here we will discuss the energy spectra for gamma rays, $\pi^{0} \mathrm{~s}$ and neutrons from the obtained data. In addition we will also discuss the prospects of $13 \mathrm{TeV}$ p-p collisions as well as that for the possible future measurement of $\mathrm{p}$-p or $\mathrm{p}$-light ions collision data in the very forward region at RHIC or at $\mathrm{LHC}$.

The 34th International Cosmic Ray Conference,

30 July- 6 August, 2015

The Hague, The Netherlands

${ }^{*}$ Speaker.

$\dagger$ 


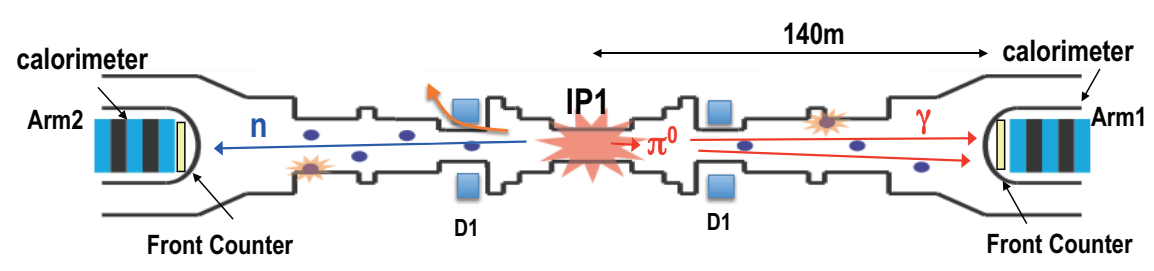

Figure 1: The schematic view of the layout of the LHCf experiment.

\section{Introduction}

Observations of ultra-high-energy-cosmic-rays (UHECRs') by extensive air showers can be interpreted on the basis of the correct modeling of hadron interactions at very high energies. Recent Large Hadron collider (LHC) experiments have provided useful data on hadron interactions at the collision energy of $10^{17} \mathrm{eV}$ in the laboratory frame. Some parameters in the hadronic interactions of cosmic rays, such as inelastic cross sections, secondary particle spectra, inelasticity, primarily influence the development of air showers. The secondary particle spectra at the very forward region is an important for understanding air shower development, while there is not much data available from experiments at such high energies.

The Large Hadron Collider forward (LHCf) experiment is dedicated to measure the production of neutral particles at the very forward region of the LHC interaction point IP1. The two detectors, Arm1 and Arm2, consist of a pair of compact electromagnetic sampling calorimeters. Each sampling calorimeter has 16 layers of plastic scinitillators interleaved with tungsten plates of 2 radiation lengths. There are four layers of position sensitive detectors made with $1 \mathrm{~mm}$ square plastic scintillating fibers (Arm1) or Si strip detectors (Arm2) inserted in order to reconstruct the position of the incident particles. Consequently, rapidity or transverse momentum of the incident particles or invariant mass of two incident particles can be reconstructed. The detailed and typical performance

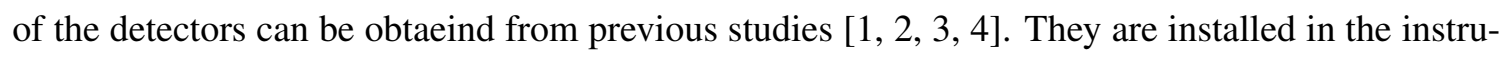
mentation slot of the Target Absorber Neutral (TAN), $140 \mathrm{~m}$ on either side of IP1 (Fig W). Here is a location of transition of a single beam pipe to two beam pipes. All the charged particles are swept out by the separation magnet (D1), and only neutral particles emitted in the pseudorapidity range, $8.6<\eta<\infty$ can be detected. This unique location is suitable to study the particle energy spectra at the very forward region for understanding air showers.

Until now LHCf experiment published data measurement for p-p collision at $\sqrt{s}=0.9 \mathrm{TeV}, 2.76$ $\mathrm{TeV}$ and $7 \mathrm{TeV}$ as well as for $\mathrm{p}-\mathrm{Pb}$ collisions at $\sqrt{s_{N N}}=5.02 \mathrm{TeV} / n$. These data have been compared with various existing cosmic ray interaction models such as SYBILL [వ], QGSJETII [G],

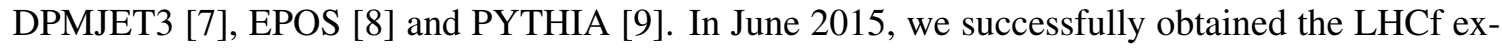
perimental data for $\mathrm{p}-\mathrm{p}$ collision data at $13 \mathrm{TeV}$. The detail of the present study on LHCf experiment along with the first look of $13 \mathrm{TeV}$ data is presented in this paper. In addition future prospects will also be discussed.

\section{Current results from $\mathrm{LHCf}$}

The measurements of energy spectra for single gamma rays at $7 \mathrm{TeV}$ [ए]] and $0.9 \mathrm{TeV}$ [प]] 

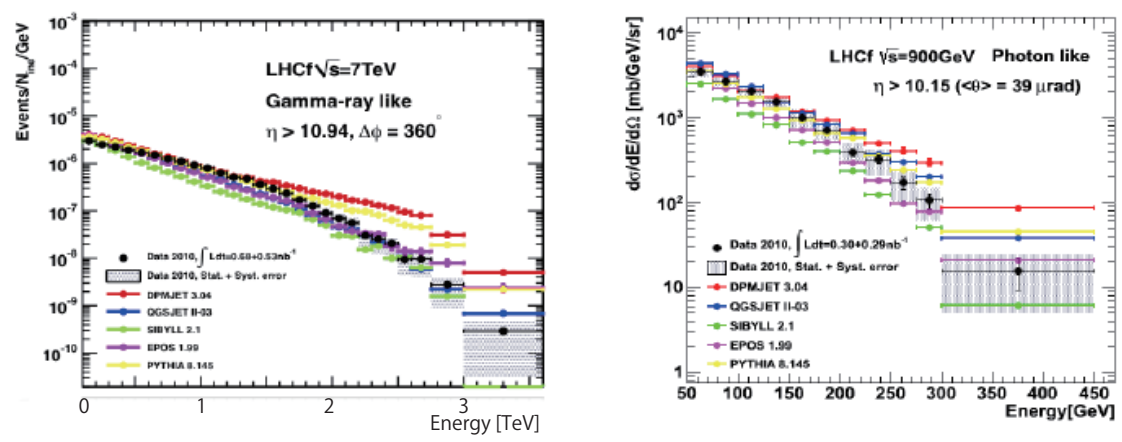

Figure 2: The very forward gamma ray energy spectra in the pseudorapidity region $\eta>10.94$ for $7 \mathrm{TeV} p-p$ collisions (left) [ए]] , and in the pseudorapidity region $\eta>10.15$ for $0.9 \mathrm{TeV}$ p-p collisions (right) [ए]]. The black histograms show data with systematic errors represented by hatched boxes. The colored histograms show the expected distributions simulated with DPMJET3.04 (red), QGSJET II-03 (blue), SYBILL 2.1 (green), EPOS 1.99 (purple), PYTHIA 8.145 (yellow).

p-p collisions have been reported. In this study, single gamma rays are dominantly generated from $\pi^{0}$ decays produced in the very forward region. Figure $\square$ shows gamma ray energy spectra for 7 $\mathrm{TeV}$ p-p (left) and $0.9 \mathrm{TeV}$ p-p (right). As shown in Fig. \(left), the energy spectra obtained by DPMJET or PYTHIA (with default parameters) show harder than that of data. The spectra for QGSJET II , EPOS and SYBILL show the shape as similar as that of the data, while the production yields for SYBILL is almost a half of the data. This tendency would be mostly similar for the 0.9 $\mathrm{TeV}$ data. Although none of them could completely reproduce the data, the experimental data are well bracketed by the expected spectra obtained by these models. This implies the systematic errors in the air shower measurement estimated for the difference between different interaction models should be appropriate.

The LHCf detector can efficiently discriminate between electromagnetic and hadronic showers on the basis of the longitudinal profile of showers. Hadronic showers discriminated from gammaray events have been also reconstructed to measure the very forward neutron energy spectra. Since the LHCf detector does not have sufficient hadronic interaction length $\lambda=1.6$, the energy resolution for hadronic showers is relatively worse $(\sim 40 \%)$. Nevertheless the energy spectra for the hadronic showers have been reconstructed using the spectrum unfolding method based on the calibration of the detector response for hadronic showers at CERN-SPS [12]]. Figure [ $]$ shows the energy spectra in the two pseudorapidity bins, $\eta>10.76$ and $8.99<\eta<9.22$, along with those predicticted by

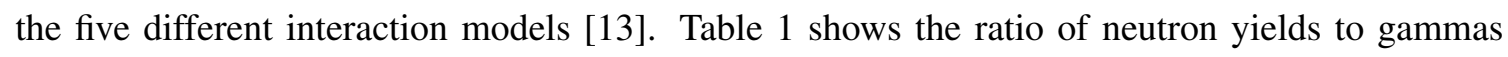
yields integrated over all energy bins for each pseudorapidity bin. The data shows the maximum neutron yields relative to the gamma yields by comparing the results obtained for the five interaction models. In the most forward pseudorapidity region $(\eta>10.76)$, the data show the maximum neutron yield with a possible bumpy structure at the higher $X_{F}$ region. As shown in Fig. B], some of the other models may show a similar $X_{F}$ shape, but they generally produce smaller neutron yields except for QGSJET II-03. QGSJET II-03 shows characteristic increase at the higher $X_{F}$ and gives the maximum neutron yield relative to that of gamma rays, as shown in Table $\square$. On the other hand, the spectrum in the smaller pseudorapidity region $(8.99<\eta<9.22)$ is well reproduced by 

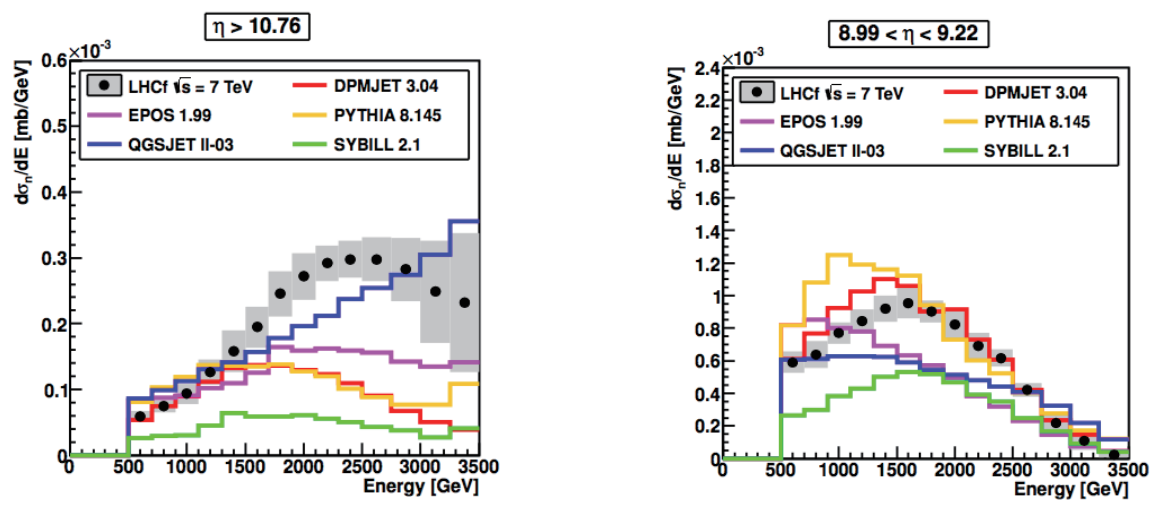

Figure 3: The neutron energy spectra in $\eta>10.76$ (left) and in $8.99<\eta<9.22$ (right) [ए]3]. The dots with hatched boxes show data with their total errors. The colored histograms show the expectation simulated by DPMJET 3.04 (red), EPOS 1.99 (purple), PYTHIA 8.145 (yellow), QGSJET II-03 (blue) and SYBILL 2.1 (green).

\begin{tabular}{|c|c|c|}
\hline & $\eta>10.76$ & $8.99<\eta<9.22$ \\
\hline \hline data & $3.05 \pm 0.19$ & $1.26 \pm 0.08$ \\
\hline DPMJET 3.04 & 1.05 & 0.76 \\
EPOS 1.99 & 1.80 & 0.69 \\
PYTHIA 8.145 & 1.27 & 0.82 \\
QGSJET II-03 & 2.34 & 0.65 \\
SYBILL 2.1 & 0.88 & 0.57 \\
\hline
\end{tabular}

Table 1: The ratio of neutron yields to gamma yields in the pseudorapidity regions $\eta>10.76$ (left) and $8.99<\eta<9.22$ (right) for data and those predicted by five different interaction models [ए[3].

DPMJET 3.04. PYTHIA also shows a relatively better agreement. These features indicate that the neutron production at zero degree may require a mechanism that is not considered in the existing interaction models ( except QGSJET II, likely ).

The transverse momentum of neutral pions in the very forward region has been measured for $\mathrm{p}-\bar{p}$ collisions at $\sqrt{s}=630 \mathrm{GeV}$ [ए4]. The measurements of $\mathrm{p}-\mathrm{p}$ collision at $\sqrt{s}=7 \mathrm{TeV}$ in the LHCf has also been reported [[5]]. Here, the transverse momentum distributions are measured for six different rapidity bins, $8.9<y<11.0$. None of the models perfectly reproduces the data distribution, while EPOS 1.99 gives a better agreement than other models. The result also shows that the data are well bracketed by the models, showing the similar conclusion as the single gamma ray samples.

The transverse momentum of neutral pions in six rapidity bins, $-11.0<y_{\text {lab }}<-8.9$, has been measured for $\mathrm{p}-\mathrm{Pb}$ collisions at $\sqrt{s_{N N}}=5.02 \mathrm{TeV}$ [ए]]. They were compared with the transverse momentum distributions obtained for $\mathrm{p}$-p collisions at the equivalent $\sqrt{s}$ deduced from the interpolation of two data samples measured at $\sqrt{s}=2.76 \mathrm{TeV}$ and $7 \mathrm{TeV}$. The nuclear modification factor 

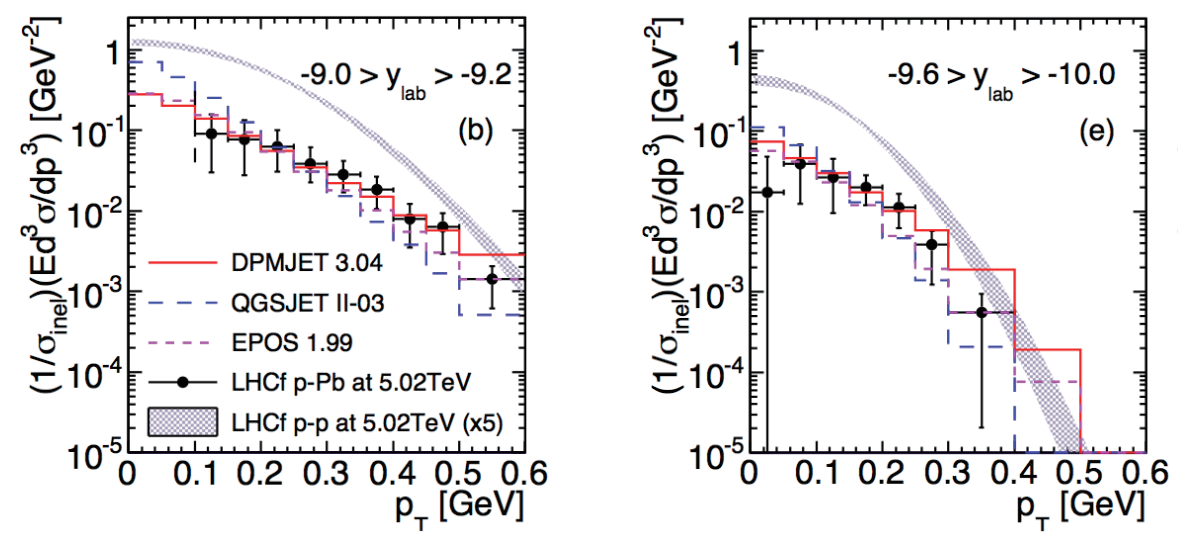

Figure 4: The $\pi^{0}$ transverse momentum distributions in the rapidity ranges $-9.0>y_{\text {lab }}>-9.2$ (left) and $-9.6>y_{l a b}>-10.0$ (right) [ए6]. The crosses represent the data and histograms show the simulated data with DPMJET 3.04 (red solid), QGSJET II-03 (blue dashed) and EPOS 1.99 (purple dotted). In addition, the expected $\pi^{0}$ transverse momentum distributions for $\mathrm{p}$-p collisions at $\sqrt{s_{N N}}=5.02 \mathrm{TeV} / n$ deduced from the p-p collision data measured at $7 \mathrm{TeV}$ and $2.06 \mathrm{TeV}$ is illustrated.

$R_{p P b}$ can be derived from the ratio :

$$
R_{p P b}=\frac{\sigma^{p p}}{\left\langle N_{b i n}\right\rangle \sigma^{p P b}} \frac{E d^{3} \sigma / d p^{3}\left(p+P b \rightarrow \pi^{0}+X\right)}{E d^{3} \sigma / d p^{3}\left(p+p \rightarrow \pi^{0}+X\right)},
$$

where $\left\langle N_{b i n}\right\rangle$ is the averaged number of binary collisions in a p- $\mathrm{Pb}$ collision, which is assumed to be $6.9 \pm 0.7$, as deduced from the Glauber theory. Figure $\$$ shows the $\pi^{0}$ transverse momentum distributions for $\mathrm{p}-\mathrm{Pb}$ collisions at $\sqrt{s_{N N}}=5.02 \mathrm{TeV} / n$ in the two rapidity bins $-9.0>y_{\text {lab }}>-9.2$ and $-9.6>y_{l a b}>-10.0$. In addition, the expected $\pi^{0}$ transverse momentum distributions are also shown for p-p collisions at $\sqrt{s}=5.02 \mathrm{TeV}$ as a denominator to calculate $R_{p P b}$. The ratio $R_{p P b}$ for the same rapidity bins is shown in Fig [5. The values of $R_{p P b}$ are about $0.1-0.3$ in the $p_{T}$ range below $0.6 \mathrm{GeV} / c$, which is almost consistent with the prediction of the interaction models QGSJET II-03, DPMJET3, and EPOS. Therefore, these cosmic ray interaction models almost correctly describe nuclear modification in the very forward region.

Reconstruction of $\pi^{0}$ is applied for an event where each gamma ray from a $\pi^{0}$ decay reaches a respective calorimeter tower in the detector ( type-I ). In this case, the acceptance for higher energy of $\pi^{0}$ is limited, as shown in Fig $\mathbf{6}$. Currently, a new "type-II" reconstruction method is being developed for an event where two gamma rays reach a single calorimeter tower [17]. The clear invariant mass peak can be reconstructed even in the type-II $\pi^{0}$ events. Combing the two types of $\pi^{0}$ events maximized the kinematical acceptance for $\pi^{0}$ and the spectra could be obtained using the full phase space.

\section{Status of $13 \mathrm{TeV}$ p-p run}

Since March 2015, LHC has started its operation with double beam energy than that used prior to the long shutdown. The LHCf experiment revisits the experimental site to obtain the p-p collisions data at $\sqrt{s}=13 \mathrm{TeV}$ in the very forward region, as we originally proposed. For this operation 

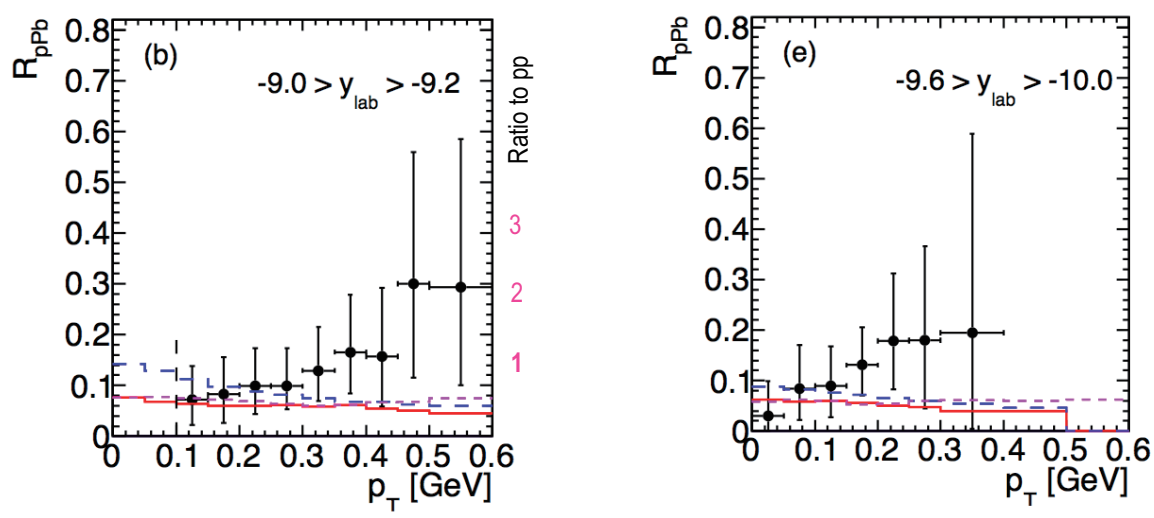

Figure 5: The nuclear modification factors $R_{p P b} \mathrm{~s}$ for $\pi^{0}$ transverse momentum distributions in the rapidity ranges $-9.0>y_{l a b}>-9.2$ (left) and $-9.6>y_{l a b}>-10.0$ (right) [ए6]. The crosses represent the data and histograms resresent the simulated data with DPMJET 3.04 (red solid), QGSJET II-03 (blue dashed) and EPOS 1.99 (purple dotted).
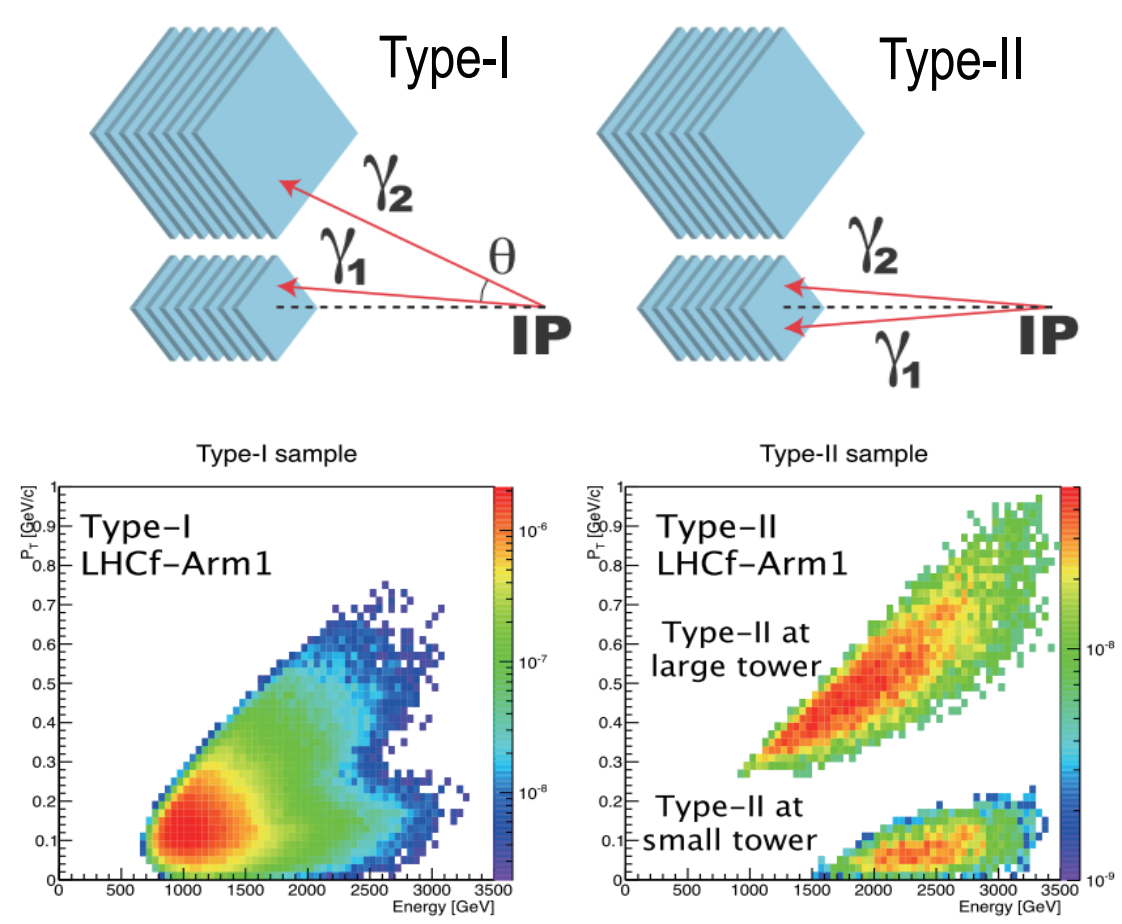

Figure 6: Type-I (left) and type-II (right) $\pi^{0}$ reconstruction methods. The two plots (below) show the momentum and $p_{T}$ acceptance of reconstructions. 

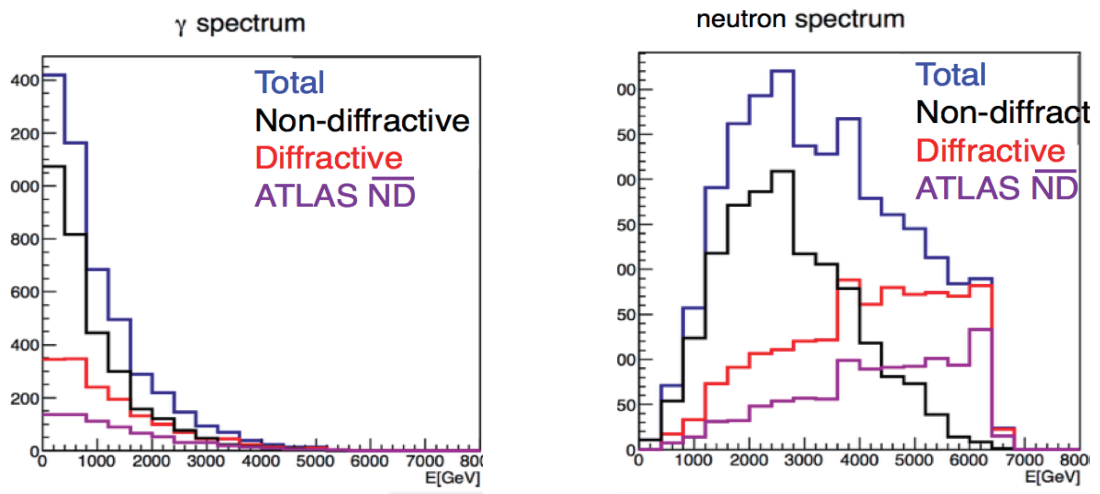

Figure 7: The expected energy distributions of gamma rays ( left ) and neutrons ( right ) detected by the LHCf detector for $13 \mathrm{TeV}$ p-p collisions simulated by PYTHIA. The red and black histograms represent the diffractive and the non-diffractive components, respectively. The purple histogram represents the events selected by non-diffractive tagging using ATLAS $N_{t r k}$ information.

we have upgraded the detectors and triggers and is discussed in detail in the following points.

First, we have replaced all the plastic scintillator components by radiation-hard crystal scintillator GSO. GSO is an excellent scintillator because of several advantageous feature, such as fast decay time, large light yields and high radiation tolerance. We have developed very thin GSO plates and a hodoscope consisting of $1 \mathrm{~mm}$ square GSO bars. We have also replaced the acrylic light guide for scintillator layers by the one made with quartz. The basic performances, such as light yields or cross talk and radiation tolerance, have been studied using heavy ion beams at HIMAC [ए8]].

Second, we have improved the trigger latency in order to cope with common data taking with ATLAS. In 2013, the pilot operation of the common data taking mode was successfully implemented for $\mathrm{p}-\mathrm{Pb}$ run. During the period, tagging diffractive events by using central information obtained using the ATLAS central detector is planned. Figure $\square$ shows the expected energy distributions for gamma ray ( left ) and neutron ( right ) at LHCf simulated by PYTHIA. The two components for diffractive and non-diffractive events are shown seprately. Here $N_{t r k}$ is the number of tracks with $p_{T}>100 \mathrm{MeV} / c$ in the rage $|\eta|<2.5$ detected in the ATLAS central tracker. We can efficiently tag non-diffractive events by requiring $N_{t r k} \geq 2$. The purple histograms in Fig. $\square$ show the events selected by diffractive tagging by using the above mentioned criteria. Using these criteria, the diffractive events can be selected with almost $50 \%$ efficiency where a few percentage is contaminated by the non-diffractive interactions. Using the data, we can study diffractive and non-diffractive components individually.

In Dec 2014, the LHCf detector was installed again in the TAN and commissioning began. In the 2nd week of Jun 2015, a special low luminosity run was dedicated for LHCf data measurement. In total 34 hours of beam fills, almost $40 \mathrm{M}$ single showers and $5 \mathrm{M} \pi^{0}$ were obtained. The detector was dismantled from the TAN during the first technical stop in the 3rd week of June. On evaluating the measured data, peaks of $\pi^{0}$ and $\eta$ were clearly observed. The detailed analysis of the data measured for the $13 \mathrm{TeV}$ p-p collision is in progress. 


\section{Future prospects}

The LHCf experiment has successfully completed the first proposed program. Besides the ongoing data analysis of obtaining collision data, we plan to have another opportunity to measure data from the two contexts discussed as follows. The first is to understand collision energy dependence and to test the Feynman $X_{F}$ scaling violation. This will be achieved by studying of LHC p-p collision data at different energies, $\sqrt{s}=0.9,2.76,7$ and $13 \mathrm{TeV}$, while the $p_{T}$ acceptance changes according to the collision energy. Relativistic Heavy Ion Collider (RHIC) also has a similar zero degree detector location as LHC, and can provide ( even polarized ) p-p collisions at $\sqrt{s}=500$ $\mathrm{GeV}$ with similar $p_{T}$ coverage as LHC because of much closer distance from IP. Measurement of data with one of the LHCf detectors for $500 \mathrm{GeV}$ p-p collision is planed in 2017 at RHIC and is currently under discussion. Ultimately Future Circular Collider with proton beam option will serve an interesting opportunity to measure the data at $5 \times 10^{18} \mathrm{eV}$ for cosmic rays.

The second point is to understand nuclear effect of cosmic ray interactions in air showers. Currently the $\mathrm{p}-\mathrm{Pb}$ collision data is available, while it is interesting to study proton-nitrogen or iron-nitrogen collisions at the high energy colliders. RHIC would provide possible proton-light ion collision data, such p-C or p-Al. In addition it is technically feasible to have possible proton - oxygen collisions at LHC. In the future, data measurements such as LHCf in the very forward region are essential to explore the cosmic ray interactions at ultra high energies.

\section{References}

[1] O.Adriani et al. [LHCf collaboration], JINST 3, S08006 (2008).

[2] T.Sako et al. [LHCf collaboration], Nucl. Instr. Meth. A578, 146 (2007).

[3] T.Mase et al. [LHCf collaboration], Nucl. Instr. Meth. A671, 129 (2012).

[4] O.Adriani et al. [LHCf collaboration], Int. J. Mod. Phys. A28, 1330036 (2013).

[5] E.J. Ahn et al., Phys. Rev. D80, 094003 (2009).

[6] S. Ostapchenko, Phys. Lett. 636, 40 (2006).

[7] S. Roesler, R. Engel and J. Ranft, Proc. of 27th Int. Cosmic Ray Conf. 2002, 439.

[8] T. Pierog and K. Werner, Phys. Rev. Lett. 101, 171101 (2008).

[9] T. Pierog and K. Werner, Phys. Rev. Lett. 101, 171101 (2008).

[10] O.Adriani et al. [LHCf collaboration], Phys. Lett. B703, 128, (2011).

[11] O.Adriani et al. [LHCf collaboration], Phys. Lett. B715, 298 (2012).

[12] K.Kawade et al., JINST 9 P03016 (2014).

[13] O.Adriani et al. [LHCf collaboration], arXiv:1503.03505, submitted to Phys. Lett. B.

[14] E. Paré et al. [UA7 collaboration], Phys. Lett. 242, 531 (1990).

[15] O.Adriani et al. [LHCf collaboration], Phys. Rev. D86, 092001 (2012).

[16] O.Adriani et al. [LHCf collaboration], Phys. Rev. C89, 065209 (2014).

[17] O.Adriani et al. [LHCf collaboration], CERN-PH-EP-2015-201.

[18] K.Kawade et al., JINST 6 T09004 (2011). 